\title{
RON HUBBARD : LE FOU, SON CORPS ET SON DÉLIRE
}

Thierry Lamote

\author{
ERES | « L'en-je lacanien »
}

2013/2 $n^{\circ} 21 \mid$ pages 141 à 154

ISSN 1761-2861

ISBN 9782749239699

Article disponible en ligne à l'adresse :

http://www.cairn.info/revue-l-en-je-lacanien-2013-2-page-141.htm

\section{Pour citer cet article :}

Thierry Lamote, "Ron Hubbard : le fou, son corps et son délire », L'en-je lacanien 2013/2 (n²1), p. 141-154.

DOI 10.3917/enje.021.0139

Distribution électronique Cairn.info pour ERES.

(C) ERES. Tous droits réservés pour tous pays.

La reproduction ou représentation de cet article, notamment par photocopie, n'est autorisée que dans les limites des conditions générales d'utilisation du site ou, le cas échéant, des conditions générales de la licence souscrite par votre établissement. Toute autre reproduction ou représentation, en tout ou partie, sous quelque forme et de quelque manière que ce soit, est interdite sauf accord préalable et écrit de l'éditeur, en dehors des cas prévus par la législation en vigueur en France. Il est précisé que son stockage dans une base de données est également interdit. 


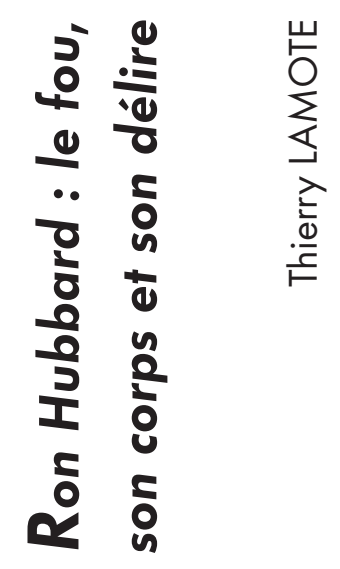

(1) a thèse freudienne est bien connue : dans la paranoïa, le délire, que l'on prend à tort pour la maladie parce qu'il « attire à grand bruit notre attention ' $"$, est en réalité une « tentative de guérison », une "solution de compromis ${ }^{2}$ ", qui non seulement rend la réalité de nouveau vivable pour le sujet, mais lui ouvre en outre une voie de retrouvailles avec ses objets - soit autant de raisons pour lesquelles les fous « aiment leur délire comme ils s'aiment eux-mêmes ${ }^{3}$ ». Si la clinique confirme cette fonction autothérapeutique du délire, les modalités de cette autothérapie restent bien souvent opaques : comment le sujet psychotique parvient-il, par le langage et l'écriture, à traiter les affres de la maladie? Suivons la trace et les progrès de ce travail du délire dans les écrits de Ron Hubbard, le fondateur de l'Église de scientologie, dont le système délirant, procédant de désordres hypocondriaques inauguraux, colla aux revirements et

Thierry Lamote est psychologue clinicien, docteur en psychopathologie clinique et psychanalyse, chercheur associé au LCPI, EA 4591, université de Toulouse 2 le Mirail.

1. S. Freud (1911), "Remarques psychanalytiques sur l'autobiographie d'un cas de paranoïa », dans Cinq psychanalyses, Paris, PUF, 1997, p. 315.

2. Cf. notamment S. Freud, La naissance de la psychanalyse, Paris, PUF, 2009, p. 135.

3. Ibid., p. 101. 
inflexions de sa maladie pour finir par s'épanouir dans ce qui deviendra la doctrine de son mouvement sectaire.

\section{L'hypocondrie, alternative ou prémisse de l'efflorescence du délire?}

Jusqu'en 1950, Lafayette Ronald Hubbard était un écrivain américain de science-fiction, connu et prolifique. En avril 1950, pourtant, après avoir publié «Greed »dans Astounding Science Fiction, il interrompt brutalement sa foisonnante activité littéraire. Dès le mois suivant paraît Dianetics, the Modern Science of Mental Health, la pierre angulaire du double système (dianétique puis scientologie) auquel il va se consacrer exclusivement jusqu'au début des années 1980.

Cette monumentale œuvre " théorique » est infiltrée et tramée par des éléments qui semblent référer à une expérience intensément douloureuse. Quid de cette douleur, qui paraît former le soubassement empirique de la doctrine? Une remarque de Freud est faite pour nous rendre sensibles à cet indice clinique. Dans son travail sur Schreber ${ }^{4}$, évoquant les nombreuses « idées délirantes hypocondriaques » du président, il indique dans une note de bas de page : "Je ne veux pas laisser passer l'occasion de faire observer ici que je ne saurais tenir pour valable aucune théorie de la paranoïa qui n'inclurait pas les symptômes hypocondriaques presque toujours concomitants de cette psychose ${ }^{5}$. "Comment entendre cette précision?

Lorsqu'il engage le praticien à être vigilant à l'égard des symptômes hypocondriaques de la paranoïa, Freud reste fidèle à la psychiatrie du $X I X{ }^{e}$ siècle, qui avait de longue date établi les liens entre l'hypocondrie et la psychose. L'on n'en devina toutefois pas immédiatement la prééminence : Jean-Pierre Falret, par exemple, se contente en 1850 de l'évoquer au passage, dans les arrière-plans $d^{\prime}$ autres perturbations. Ce qu'il repère dans la première période des troubles (période " $d^{\prime}$ incubation ${ }^{6}{ }^{\prime}$ )

4. S. Freud, "Remarques psychanalytiques sur l'autobiographie d'un cas de paranoïa», op. cit., p. 303.

5. Ibid. (note 2).

6. J.-P. Falret, Des maladies mentales et des asiles d'aliénés, rééd. Chilly-Mazarin, coll. «Sciences en situation », 1994, p. 193. 
renvoie plutôt à la mélancolie : anxiété, préoccupation et « curiosité inquiète » assaillent le malade "sans motif ${ }^{7}$ ». Le mal et la souffrance sont ainsi définis moins en relation avec le corps qu'avec le moral, quoique la dimension physique du mal ne soit pas absente dès les prémisses de la folie : « Il est aussi très fréquent, écrit-il, que quelques souffrances physiques se joignent au trouble naissant du moral ${ }^{8}$. „ À l'inverse, Morel décrit en 1860 un délire de persécution dont la spécificité est son soubassement hypocondriaque : "Pour Morel, commente Valentin Magnan, il faut qu'ils [les persécutés] soient hypocondriaques $d^{\prime}$ abord ${ }^{~}$. " La séquence morélienne du développement de la maladie se déroule ainsi en un enchaînement de trois phases : hypocondrie, persécution, ambition. Interrogeant la description de Morel d'une évolution en trois temps, Magnan y opposera une entité clinique, le "délire chronique à évolution systématique », dont la «marche méthodique, régulière et progressive » suit un développement réglé selon quatre périodes : d'incubation, de persécution, ambitieuse et de démence. En dépit de ses critiques du découpage de Morel, Magnan constate néanmoins qu'à l'orée de l'explosion de leur délire, ses malades "pourraient être pris pour des hypocondriaques ${ }^{10}$ ».

On voit donc combien les auteurs, quels que soient par ailleurs leurs points de divergence, s'accordèrent relativement uniformément à repérer un moment d'invasion de douleurs hypocondriaques lors de l'éclosion de la psychose. Freud lui-même, dans chacune des avancées de sa théorie de la psychose, portera une attention particulière à la question de l'hypocondrie : avatar du retrait libidinal spécifique à la psychose, I'hypocondrie apparaît pour lui comme alternative ou prémisse de l'efflorescence du délire. Voyons cela à l'aune de la théorie des nœuds borroméens de Jacques Lacan.

7. Ibid., p. 147.

8. Ibid., p. 312

9. V. Magnan, Le délire chronique à évolution systématique, rééd. Paris, L'Harmattan, 1998, p. 13.

10. Ibid., p. 29. 


\section{Approche borroméenne du corps...}

Partons des trois ronds du symbolique, de l'imaginaire et du réel, dont Lacan nous dit qu'ils forment les « registres essentiels de la réalité humaine " ". Pour que le sujet et sa réalité trouvent une certaine consistance, ces trois dimensions doivent être novées en une chaîne "borroméenne ", où réel, symbolique et imaginaire, libres deux à deux, sont liés en une figure ternaire par l'entrelacs du troisième terme (figure $1^{12}$ ) - ce qui fait que si l'on dénoue l'un des éléments, l'ensemble se défait (figure $2^{13}$ ):

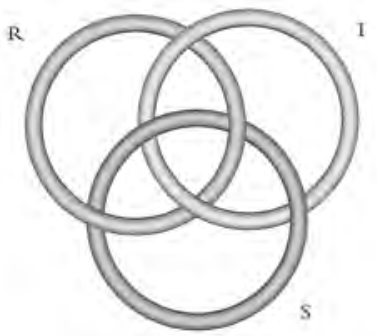

Figure 1

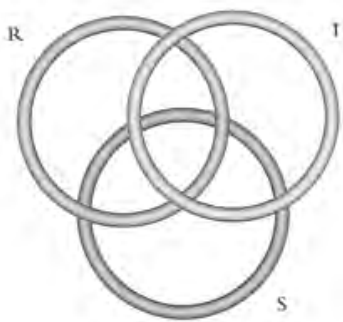

Figure 2

Cette triade RSI, absente de la théorie freudienne, Lacan affirme que Freud en eut le soupçon - à ceci près que non seulement réel, imaginaire et symbolique ne s'inscrivent qu'en filigrane dans sa réflexion, mais, de surcroît, ils y apparaissent généralement déliés, indépendants les uns des autres ${ }^{14}$ (fig. 2). Ce qui retient son attention, c'est qu'ils ne partent pas à la dérive, quelque chose les retient ensemble : une ébauche de novage se profile dans ce que Freud nommait "réalité psychique », laquelle est dans l'étroite dépendance du complexe d'OEdipe. Celui-ci, qui donne son assiette au sujet, apparaît donc comme l'instance nodale

11. J. Lacan, "Le symbolique, le réel et l'imaginaire », dans Les Noms-du-Père, Paris, Seuil, 2007, p. 13.

12. Cf. J. Lacan, Le séminaire, Livre XXIII, Le sinthome, Paris, Seuil, 2005, p. 20.

13. Ibid.

14. P. Skriabine, «Clinique de la suppléance », Ornicar?, $n^{\circ} 44,1988$. 
de la théorie freudienne, "le quatrième terme qui fait nouage des trois ronds libres $\mathrm{R}, \mathrm{S}$ ef $\mathrm{I}^{15}$ » (fig. $3^{16}$ ) :

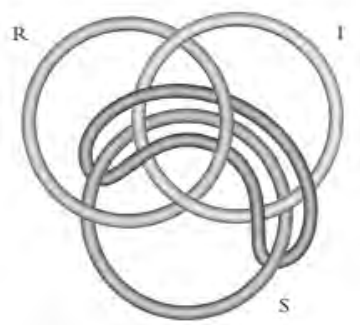

Figure 3

Le complexe d'OEdipe réussit donc ce que le nœud borroméen à trois ronds parvient à accomplir : il arrête la dérive des trois dimensions RSI en les coinçant ensemble. Cette chaîne à quatre termes est donc structuralement équivalente à la chaîne borroméenne ternaire (chaîne idéale, prototype du nouage borroméen). Or qu'est-ce qui s'infiltre, via l'OEdipe, pour y produire un effet de nouage, sinon la fonction paternelle ? « Le complexe d'OEdipe, précise Skriabine, le quatrième rond, est donc un Nom-du-Père ; et aussi bien, le nouage borroméen en tant que tel, comme quatrième, est un Nom-du-Père ${ }^{17}$. " Nous y voilà : si le quatrième terme du nouage à quatre, à l'œuvre dans et par l'OEdipe, est l'un des Nomsdu-Père, la chaîne à trois ronds, qui tient toute seule, est donc en ellemême un Nom-du-Père. Mais le nouage par le Nom-du-Père, ce quart élément issu du complexe d'OEdipe qui est susceptible de venir suppléer au fonctionnement idéal de la chaîne à trois, n'est pas la seule solution : le symptôme, en mordant chacune des dimensions RSI, peut également offrir un aménagement à la structure subjective de façon à ce que les trois ronds, coincés ensemble, posent un cadre à l'objet a ll'objet manquant, cause du désir), tout en venant d'une part limiter les dérives du sens et d'autre part border et domestiquer la jouissance en la réglant sur le symbolique ${ }^{18}$.

15. Ibid.

16. J. Lacan, Le séminaire, Livre XXIII, Le sinthome, op. cit.

17. P. Skriabine, "Clinique de la suppléance», op. cit.

18. J. Lacan, Le séminaire, Livre XXIII, Le sinthome, op. cit., p. 48-57. 
Voilà pour ce qui se produit sur le versant de la névrose. Côté psychose, en revanche, le nœud est défait, ce qui n'enlève pas au sujet la possibilité de tenir et de cheminer en compensant ce défaut (notamment en usant d'identifications imaginaires, "par une sorte d'imitation extérieure ${ }^{19}$ ") - du moins jusqu'à ce que, "à un certain carrefour de son histoire biographique ${ }^{20}$ », il y soit confronté et que volent en éclats les mécanismes de compensation qui l'avaient soutenu jusqu'alors. L'on sait qu'il est possible, en amont de la décompensation, de discerner les indices cliniques d'une défaillance du nouage RSI. Intéressons-nous tout de suite à l'un de ces indices (plus ou moins) discrets de la structure psychotique : I'inertie libidinale, que l'on trouvera dans un instant au cœur de la scientologie, et qui intéressa Colette Soler dans son texte « Autisme et paranoïa ${ }^{21}$ ».

\section{... et de ses désordres psychotiques : débranchement et délocalisation de la jouissance...}

À propos de Stanley, I'enfant autiste observé par Margaret Mahler, Soler note qu'il se branche sur l'Autre - corps de l'analyste, corps du signifiant - , ou se débranche. Lorsque l'enfant se branche, touché par un signifiant ou touchant l'analyste, "il se réveille et se machinise ${ }^{22}$ »: son corps s'anime de mouvements d'automate. Mais s'il se débranche, son corps tombe inerte, désarticulé, "c'est une sorte de paquet de chair ». Par « inerte », il nous faut entendre « inerte libidinalement», puisque son corps comme organisme n'est pas en panne et continue à fonctionner. Tout se passe comme s'il « avait lui-même une sorte de choix entre se brancher sur l'Autre ou pas. Nous pouvons écrire cela avec la formule de l'aliénation : ou bien il est un pur vivant, sans libido, au sens du désir, inerte donc, ou bien il devient une machine signifiante, il est machinisé ${ }^{23}$ ». L'on doit donc ici séparer le corps vivant du sujet et le corps de l'Autre : quand il se branche sur l'Autre, le corps du signifiant vient empiéter sur le vivant et

19. J. Lacan, Le séminaire, Livre III, Les psychoses, Paris, Seuil, 1981, p. 285.

20. Ibid., p. 229.

21. C. Soler, "Autisme et paranoïa », dans L'inconscient à ciel ouvert de la psychose, Toulouse, PUM, 2002, p. 63-80.

22. Ibid., p. 75.

23. Ibid., p. 76. 
crée « une zone d'incidence où le signifiant gouverne le corps » - c'est dans cette interaction des deux corps qu' « il faut situer tous les phénomènes où le corps se présente comme mécaniquement animé ».

Le problème des frontières du corps du psychotique, qui a la sensation de fusionner avec ses objets, peut alors s'éclairer : lorsque la zone $d^{\prime}$ incidence du signifiant, qui anime libidinalement le vivant, est plus étroite que l'organisme, « inversement, l'Autre apparaît comme un prolongement libidinal ${ }^{24}$ ». Ce problème tient donc moins à une « confusion de frontières imaginaires » du corps qu'à une confusion « quant au point d'insertion de la libido »: il s'agit non pas d'un défaut de perception, mais de perturbations de "l'instrument libido ». Tout se passe ici «comme si leur inclusion dans l'Autre du signifiant se traduisait au niveau du corps par le fait que la libido soit aussi de l'Autre ${ }^{25}$ ». La problématique de la séparation s'explique dans le même fil : le sujet « ne peut pas se séparer de l'Autre parce que l'Autre n'est pas un objet compensateur de son manque mais une partie de lui. Qu'il s'en sépare et son corps tombe inerte ${ }^{26}$ ». Le sujet est ainsi condamné à être soit " marionnette de l'Autre», avec lequel il se confond, soit «pur réel ${ }^{27}$ ». Ne trouvons-nous pas ce binaire au fondement de la dianétique ${ }^{28}$ - premier temps du système délirant élaboré par Ron Hubbard?

\section{... et leur traitement délirant}

Un postulat majeur fonde la dianétique : tout ce que fait l'être parlant, tous ses actes, toutes ses pensées et émotions lui sont dictés à la lettre

24. Ibid., p. 78.

25. Ibid.

26. Ibid., p. 79.

27. Ibid., p. 80.

28. La dianétique, sorte de méthode de développement personnel inspirée des Études sur I'hystérie, se fonde sur une topologie originale du psychisme humain, le « mental », qui se divise en trois parties : le "mental somatique», partie archaïque du cerveau soumise au «mental analytique » ou au « mental réactif »; le « mental analytique », " calculateur », tout-puissant, qui pilote l'homme selon la loi de la « survie » (en évitant la douleur) et enregistre toute sa vie, avec tous ses détails, en « archivant » chaque souvenir dans les «banques mnémoniques standard » par catégorie de perception (" perceptics»); et le «mental réactif », qui prend les commandes lorsque surviennent douleur et [suite page suivante] 
par ses « engrammes ${ }^{29}$ ». Ces derniers, fonctionnant comme les programmes informatiques des ordinateurs, dédouanent ainsi l'individu de toute responsabilité dans ce qui lui arrive puisque, lorsqu'un engramme est "restimulé », il déconnecte l'individu et « prend les commandes de l'organisme » pour «dramatiser »- rejover, mettre en scène - les données qui sont contenues en lui ${ }^{30}$.

Parmi les différents engrammes, Hubbard met particulièrement en garde contre ce qu'il nomme l'« engramme de la compassion ${ }^{31}$ » né de la sollicitude de l'environnement du sujet qui le plonge dans un intolérable état de dépendance et de soumission -, qu'il tient pour responsable de «toutes les maladies psychosomatiques chroniques sans exception ${ }^{32}$ ». Ces personnes élues sur lesquelles le sujet se branche sont nommées «alliés ${ }^{33}$ ». Suivons la définition - néologique et teintée de transitivisme - qu'il nous en donne : "En Dianétique, ["allié"] signifie fondamentalement quelqu'un qui protège une personne en état de faiblesse et qui en vient à avoir une influence très forte sur cette personne. La personne faible, un enfant par exemple, prend les mêmes caractéristiques que l'allié ${ }^{34}$. »

Que se passe-til quand cet « allié » vient à disparaître ? Ron Hubbard, pour y répondre, improvise une ingénieuse théorie du « débranchement »: "Toute perte grave [...] "endigue" l'émotion dans un engramme. Une perte peut créer un choc si puissant que l'analyseur [i.e. la conscience, la capacité à répondre rationnellement aux situations] se trouve hors service. Un engramme s'installe alors. S'il s'agit de la perte d'une personne [l'allié] qui a manifesté de la compassion et dont l'individu a été dépendant, celui-ci a soudain l'impression que la mort le guette. Lorsque le

inconscience : les sensations qui constituent les souvenirs ne sont alors plus classées par " perceptics», mais amalgamées dans l'engramme.

29. Traces des traumatismes douloureux s'imprimant directement dans les cellules du corps. 30. Cf. L. R. Hubbard, La dianétique, La puissance de la pensée sur le corps, Copenhague, New Era Publication International ApS, 2003, p. 101.

31. Ibid., p. 137.

32. Ibid.

33. Ibid., p. 206-207.

34. Ibid., p. 207. 
réducteur se présente sous la forme d'une perte, c'est comme si un puissant ressort était comprimé à l'intérieur de l'engramme. Lorsqu'il se détend, un torrent d'émotion est libéré $[. . .]^{35}$. » Autrement dit : lorsque l'« analyseur » est mis " hors service », à savoir lorsque l'Autre défaille, le corpsmachine, après un vif débordement de jouissance, voit sa « force vitale [...] dégringoler », tandis que l'individu tombe, désanimé, "laissé en plan » (pour le dire avec Schreber ${ }^{36}$ ). L'homme, alors, «meurt. II cesse de bouger ou de penser. II meurt en tant qu'organisme. Puis il meurt en tant qu'agrégat de colonies cellulaires. Les périodes de "vie après la mort" varient chez les cellules. [...] Donc, la mort suit plusieurs phases: tout d'abord l'organisme, puis les cellules, colonies par colonies ${ }^{37}$ ". Que sont ces cellules qui ne meurent pas en même temps que l'organisme, mais disparaissent en un temps second, « colonies par colonies»? Ici s'introduit, en catimini, le support essentiel des conceptions délirantes de Ron Hubbard : la cellule.

La cellule convoquée dans la dianétique est une entité pensante, dotée d'une certaine forme d'intelligence ${ }^{38}$. Grégaire et éminemment sensible au moindre choc, elle tend à s'associer en colonnies, et ce sont ces colonies de cellules « qui gardent l'expérience des engrammes ${ }^{39}$ ». Les cellules emmagasinent ainsi à la fois les coups reçus et les mots qui les accompagnent. D'ailleurs, à bien lire Hubbard, l'on doit concevoir qu'elles n'emmagasinent pas, à proprement parler, les chocs et les traces verbales : elles sont elles-mêmes ces traces. Elles ne possèdent donc pas la consistance matérielle d'un élément de l'organisme, leur substance est d'un autre registre : le mot « cellule » ne renvoie chez Hubbard à rien qui relèverait de la science - c'est un signifiant néologique, sans lien avec la biologie. Fondamentalement immatérielle, cette cellule-ci est un composé de jouissance et de langage. Plus exactement : elle est l'élément par lequel le langage imprime ses effets de jouissance dans l'organisme.

35. Ibid., p. 152.

36. Cf. D. P. Schreber, Mémoires d'un névropathe, Paris, Seuil, 1985, p. 61.

37. Ibid., p. 142.

38. L. R. Hubbard, La dianétique, La puissance de la pensée sur le corps, op. cit., p. 91.

39. Ibid. 
Substance signifiante, la cellule se distingue ainsi du corps organique, auquel elle ne semble raccordée que par l'énigmatique «force vitale».

Ne peut-on voir dans cette «force vitale » décrite par Hubbard, qui fait tenir ensemble le corps organique avec cet autre corps, ce « corps du langage " composé de colonies cellulaires qui sont autant de molécules signifiantes, l'intuition de ce qui lui manque pour que tiennent ensemble le corps (I), le logos (S) et le vivant (R), à savoir « la fonction du signifiant phallique ${ }^{40}$ qui conjoint le logos au vivant ${ }^{41}$ »? « La force vitale, écrit-il, est l'hélium qui gonfle l'enveloppe du ballon et lui permet de voler. Si l'hélium s'échappe, le ballon s'écrase ${ }^{42}$. "Traduisons: lorsque la «force vitale ", c'est-à-dire le signifiant phallique, est en place, le corps organique, nové au corps du signifiant, s'anime. Mais lorsque manque le signifiant phallique, l'ensemble se dénove : le corps tombe, laissé en plan ; la chaîne signifiante se défait, libérant des signifiants déchaînés, énigmatiques, tandis que l'organisme subit les affres d'une jouissance délocalisée.

Quelques années après sa rédaction, la dianétique sera remodelée dans le cadre de la scientologie. Lors de ce passage, la structure fondamentale du thème délirant ne sera nullement modifiée - le même schéma se maintiendra, réorganisé à partir d'un nouveau faisceau de signifiants. La théorie cellulaire laissera alors place à la doctrine du " thétan »: "L'homme, écrira Hubbard, est composé d'un corps, d'un mental et de ce que nous appelons un thétan - le terme de Scientologie pour nommer l'esprit, l'individu lui-même qui vit dans le corps et le dirige. » La structure

40. Rappelons que le langage dérange le corps vivant et le vide de sa jouissance pour la localiser ailleurs, en un hors-corps vers lequel tendra la pulsion. C'est par la « métaphore paternelle » que se produit cette opération : elle introduit le sujet à l'ordre symbolique, via un décollement $d^{\prime}$ 'avec l'objet primordial de sa jouissance par où s'introduit le manque qui aiguillonnera le désir. Le signifiant phallique, " $\phi$ », résulte de la métaphore paternelle : symbolisant la perte de jouissance nécessaire à l'exercice de la parole, il désigne la fonction qui, en s'étayant sur le Nom-du-Père ou le symptôme, permet l'intrication de la jouissance (autorisée à l'être parlant) avec le corps et le langage. Cf. J. Lacan, " D'une question préliminaire à tout traitement possible de la psychose », dans Écrits, Paris, Seuil, 1966.

41. J.-C. Maleval, Logique du délire, Paris, Masson, coll. «Ouvertures psy », 2002, p. 145.

42. L. R. Hubbard, La dianétique, La puissance de la pensée sur le corps, op. cit., p. 142. 
binaire des deux corps - le corps physique et le corps du signifiant (le "thétan ») qui donne son animation libidinale au corps physique - est maintenue, précairement novée par un troisième terme, le « mental », nouvelle intuition délirante du signifiant phallique en défaut. $C^{\prime}$ est le «mental » qui est l'objet de toutes les défaillances et, partant, de toutes les attentions de la technique scientologique. Lorsque le mental ne remplit plus son office, le " thétan » se libère brutalement de sa gangue de matière, tandis que le corps s'effondre dans une invasion perturbante de « douleur ».

Hubbard ne nous donne-t-il pas, sur les deux versants de son délire, une description quasi clinique des déclenchements de sa psychose? Une théorie de l'effondrement psychotique en deux temps se discerne ainsi dès le livre un; elle se maintiendra avec une remarquable rigueur jusqu'aux dernières élaborations de la doctrine : tout d'abord, la "force vitale » (dianétique) - ou le «mental » (scientologie) -, cet équivalent délirant du signifiant phallique, se défait; puis le corps meurt, il s'effondre en une inertie catatonique qui fige les pensées du sujet, tandis que la chaîne des signifiants se désagrège, "colonies par colonies », et que la jouissance se délocalise pour s'éparpiller dans l'organisme en douleurs diffuses.

$C^{\prime}$ est vraisemblablement ce qui se produira à diverses reprises pour Ron Hubbard. À chaque fois, il se retrouvera "laissé en plan », assailli par l'intrusion massive d'un jaillissement de sensations douloureuses qui submergeront son organisme. Entre deux morts, entre la mort de l'organisme et celle des cellules, c'est-à-dire entre la mort du corps de jouissance et celle du corps du langage qui le marque et lui confère son souffle, il s'affaissera sous la forme d'un clochard - corps loqueteux, " débranché », libidinalement inanimé. Lorsque la suppléance lâche, l'enveloppe imaginaire du corps se décroche : perdant ses attaches avec le symbolique et son lien au réel de la jouissance par lequel s'insuffle la vie, le corps «fou[t] le camp », nous dit Lacan, " [i]l glisse, le rapport imaginaire $n^{\prime}$ a pas lieu ${ }^{43} »$. C'est pour cela que, après avoir décrit le débranchement et la délocalisation de la jouissance, Hubbard théorisa ce que Lacan nomma le « laisser tomber du rapport au corps propre ».

43. J. Lacan, Le séminaire, Livre XXIII, Le sinthome, op. cit., p. 151. 


\section{Une ponctuation du délire : la théorie de libération du « thétan »}

Toute la construction délirante de Ron Hubbard semble orientée par une puissante aspiration à se débarrasser du corps. Dans la dianétique, le corps («mental somatique ») est présenté comme le lieu où sont « engrammées » les douleurs subies tout au long de sa vie par l'individu : en lui gît une source de nuisance menaçant de se réveiller à tout moment. Le but de l'initiation dianétique, phase préliminaire à l'entrée en scientologie proprement dite, est l'état de « clair » : lumineux état où se libèrent les potentialités du «mental analytique», dont la puissance est bridée par le corps. Dans le deuxième livre de Ron Hubbard, Science de la survie ${ }^{44}$, le corps est un simple objet naturel parmi d'autres, l'un des éléments de ce qu'il nomme MEST ${ }^{45}$, dont l'unique singularité est d'avoir été télescopé par l'élan vital (nommé « thêta »). Le but de la thérapie dianétique se précise alors : il s'agit de libérer "thêta », de façon à ce qu'il se disjoigne de MEST pour soumettre ce dernier à sa volonté. Lorsque apparaît la scientologie, le rejet du corps s'accentue. Hubbard affirme, dans Scientologie, Une nouvelle optique sur la vie, que "les gens ne [sont] pas des corps, mais des unités vivantes faisant fonctionner des corps ${ }^{46} \gg$ : cette unité vivante, le «thêta », "c'est l'individu, la personne, l'identité réelle ${ }^{47}$ ». Or cette vraie identité est « modifiée par l'adjonction d'un corps »: c'est cette adjonction qui conduit l'identité « à une certaine méconnaissance de sa condition ${ }^{48}$ ».

La scientologie propose à chacun, à chaque «thêta » qui s'ignore, d'élever sa connaissance : qu'il sache de nouveau qui il est, de façon à appliquer à l'univers physique son incommensurable pouvoir. Une véritable guerre contre l'organisme et la matière s'engage dès les premiers balbutiements de la scientologie lorsque Ron Hubbard prophétise le « combat

44. L. R. Hubbard, Science de la survie, Copenhague, New Era Publication International ApS, 2001.

45. "Matter, Energy, Space, Time».

46. L. R. Hubbard, Scientologie, Une nouvelle optique sur la vie, Copenhague, New Era Publications International ApS, 2007, p. 27.

47. Ibid.

48. Ibid. 
de titan » qui attend ses adeptes, à savoir la lutte acharnée de «l'énergie de la vie contre la matière, l'énergie, l'espace et le temps. La vie contre l'univers physique ». En bout de course, la conquête finale, le couronnement du parcours initiatique qui polarise le désir d'innombrables scientologues, est l'état de «thétan opérant » : état idéal promettant - via des techniques qui épousent aussi bien les lignes de force de la structure psychotique de Ron Hubbard que les fantasmes biotechnologiques contemporains - de s'émanciper de son corps.

\section{Le retour du fou en position de maître}

Hubbard est donc parvenu à articuler en une doctrine porteuse de sens les phénomènes élémentaires qui l'assaillirent. Si la dianétique lui a permis d'éponger l'étrangeté et l'extrême singularité de son expérience psychotique en faisant du branchement, du débranchement et de l'hypocondrie des processus universels inhérents à la vie elle-même, ce n'est qu'au second temps, "scientologique ", de son système que surgit sa trouvaille la plus astucieuse : sa théorie de la libération du thétan. Grâce à elle, il put faire du "laisser tomber du rapport au corps », phénomène élémentaire psychotique, le point idéal vers lequel l'humanité doit s'élever pour atteindre la strate supérieure de son évolution. $\mathrm{N}^{\prime}$ a-t-il pas alors anticipé sur cette pente de la pensée technique postmoderne, laquelle - influencée par l'interprétation des « vieux refrains chantant les pouvoirs de l'esprit sur la matière ${ }^{49}$ " selon le paradigme des théories de l'information et par les avancées de la technoscience et des biotechnologies ${ }^{50}$ consiste en une puissante aspiration à "se libérer de la matière »?

Le travail délirant compilé dans ses ouvrages de dianétique et de scientologie, en phase avec son temps, a immédiatement rencontré un vaste auditoire. Par son délire, Hubbard non seulement a réussi à se réinscrire dans la communauté des hommes "ayant toute leur raison », mais de surcroît est parvenu à s'aménager une place dans le lien social : la place d'un prophète des temps nouveaux - place à part, en position

49. B. Bensaude-Vincent, Se libérer de la matière ? Fantasmes autour des nouvelles technologies, Paris, INRA éditions, 2004, p. 19.

50. Cf. D. Lecourt, Humain, Post-humain, Paris, PUF, 2003. 
d'exception, qui fit de lui le modèle à suivre pour qui se préoccupe du salut de l'humanité. Par son délire, Hubbard a donc construit de toute pièce la seule place où il était « de structure » susceptible de se soutenir dans la « cité des discours », à savoir la place du maître : pour le meilleur (sa propre stabilisation, qui le mènera, via sa réconciliation avec l'Autre - versöhnung ${ }^{51}$-, à un certain apaisement) et pour le pire (de nombreux adeptes souffrent de s'être aliénés à son système délirant).

51. S. Freud, «Remarques psychanalytiques sur l'autobiographie d'un cas de paranoïa», op. cit., p. 280. 